\title{
Assessing slide coverage by cytoscreeners during the primary screening of cervical smears, using the AxioHOME ${ }^{\circledR}$ Microscope system
}

\author{
Robert W. Baker ${ }^{1}$, Gerard Brugal ${ }^{2}$ and Dulcie V. Coleman ${ }^{1}$ \\ ${ }^{1}$ Department of Cytopathology and Cytogenetics, Imperial College School of Medicine at St Mary's, \\ Praed Street, Paddington, London W2 1NY, UK \\ ${ }^{2}$ Institut Albert Bonniot, Université Joseph Fourier, Grenoble, France
}

Received 26 June 1996

Revised 21 November 1996

\begin{abstract}
We have used the HOME Microscope system to examine the screening patterns of cytotechnologists who undertake the primary screening of cervical smears, in order to measure accuracy of screening against screening time, slide coverage, and mean screening rate. Twelve cytotechnologists engaged routinely on cervical screening volunteered for this study. They were asked to perform primary screening of 10 test slides under normal laboratory conditions in the normal way. Slide coverage and screening time were recorded on the HOME system. Slide maps were prepared and the results analysed.

The exercise demonstrated that all primary screeners fail at some point to scan the whole of the slide during primary screening. The maps produced by the HOME system clearly demonstrated that 5 different types of error can occur that lead to incomplete coverage of the slide. Mean slide coverage was $84 \%$, and some individuals averaged only $66 \%$ coverage. The results show that there is a major problem in the education of some individual cytotechnologists in slide coverage. This could be rectified by the incorporation of a HOME system into every training centre, and the establishment of a protocol for assessment of slide coverage in competence examinations. Furthermore, the exercise has shown that even those individuals who normally attain a good standard of slide coverage would be able to improve slide coverage given access to the daily use of a HOME Microscope, or a system with equivalent screening/reviewing functionality.
\end{abstract}

Keywords: HOME Microscope, cervical screening, slide coverage, screening accuracy

\section{Introduction}

In order to ensure that the whole coverslipped area of a cervical smear is observed microscopically by a cytotechnologist, it is necessary to overlap adjacent fields of view. National guidelines for microscopic screening procedures ought to reflect this fact. Regional training programmes and competence examinations should include both training in and assessment of the techniques necessary to achieve full slide coverage. In practice, however, no method of assessment has been possible to determine whether a screener does actually cover the whole slide before they are deemed competent in the examination of cervical smears. Yet one would assume that such a fundamental principle of slide examination ought to be an essential feature of any assessment of an individual's ability to examine 
cervical smears. It is certainly documented for automated screening systems, so why not for the much less accurate manual method?

The AxioHOME ${ }^{\circledR}$ system is a new development which provides the cytotechnologist with detailed feedback regarding their screening track and screening time. The microscope consists of a 486 PC computer attached to the stage via $\mathrm{x}-\mathrm{y}$ transducers and electronic sensors in the turret. The system overlays a computer field over the microscope field via a video link through a half silvered mirror in the body of the microscope. It receives continuous data relating to the size and position of the field of view which it records as co-ordinates, and continually updates the field projected through the microscope. These features allow the accurate marking and labelling of individual cells, as well as ready reference to clinical data, and easy diagnosis and reporting facilities. The system can be used to analyse the coverage of a slide and the time spent screening a slide. From these data it is possible to deduce a mean rate of screening.

The aim of this study was to assess the slide coverage by the primary screener in the routine laboratory screening environment, and to try to evaluate whether this might have any bearing on the issuing of a false negative result. In order to achieve this aim, we invited cytotechnologists from a range of nearby laboratories to attend this department and to participate in the primary screening of a set of 10 slides, and recorded the slide coverage and screening time using the AxioHOME ${ }^{\circledR}$ system.

\section{Materials and methods}

The HOME system has been described elsewhere [1,2]. It can be used to assess the slide coverage during screening, and measure the time spent during the screening process. It is also possible for checkers to review coverage of primary screeners, and rectify any deficiencies by rescreening unscreened areas. Once the scanning track and screening time are recorded, the data in the form of a map of the slide can be exported to the Paintbrush windows application, where the picture can be inverted, and colours edited ready for printing (see Fig. 1).

\subsection{Study group and test procedures}

Cytotechnologists were invited from North Thames Region cytology laboratories to participate in the primary screening of a test set of 10 slides. Volunteers were recruited initially by an invitation

a)

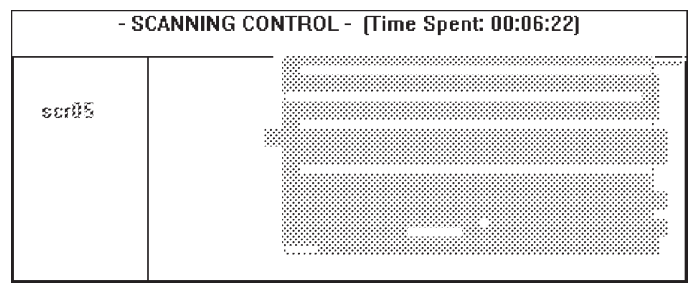

c)

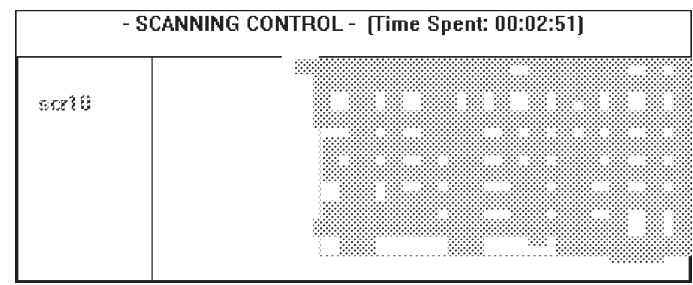

b)

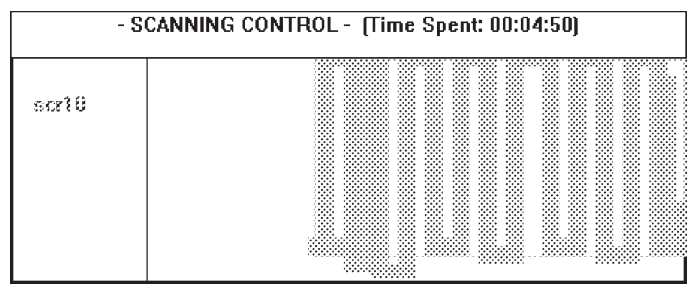

Fig. 1. (a) Horizontal screening. (b) Vertical (battlement) screening. (c) Combined screening. 
letter which was sent to consultant pathologists in laboratories in the North Thames regions, followed by a telephone call to the laboratory manager. Volunteers attended this department by appointment. All volunteers participating in the primary screening study were primary screeners who normally did screening as part of their routine workload. Ten volunteers had a certificate of competence in cervical cytology (BSCC, IBMS, IAC). Two volunteers had not passed a competence examination, but both had nine years screening experience. Screeners were not informed of the capabilities of the system, but were advised of the confidentiality of the test results.

A set of ten slides was selected, to be preceded by three "acclimatisation" slides which were included in the test set for the screener to primary screen, in order to become familiarised with the microscope controls and the test procedure. The slides were rescreened by the administrator and a pathologist to verify the original diagnosis. Only the results from the ten test slides were analysed. Slides for the primary screening study were selected as follows: five negative slides, two unsatisfactory slides, three positive slides. Statistical analysis indicated that a test set of ten or more slides screened by ten or more people would provide sufficient data to detect a significant result with type I error 5\% and power $80 \%$. Furthermore, the fact that the same set of slides was observed by every volunteer ensured that variability amongst slides and variability amonst observers would be minimised. All slides were mounted with a $22 \times 40$ coverslip. The negative slides were selected as being of adequate cellularity and representative of the cervical transformation zone. Abnormal slides were selected carefully and screened by two cytotechnologists in a double blind test, and all abnormal cell groups and single cells were recorded as electronic dots on the AxioHOME ${ }^{\circledR}$ system. A map of the abnormal cell distribution was exported via the Paintbrush application. The total number of dots was recorded.

Prior to each test procedure, the administrator cleaned the microscope eyepieces and objectives, and ensured that the microscope was set up for Kohler illumination. The stage and objectives were calibrated according to the HOME protocol, and the stage was centred using the calibration slide. Slides were cleaned, dots removed with alcohol, excess mountant removed, and bubbles were eliminated by remounting the slide. Stage tension and objective configuration were set in an identical configuration for each volunteer, the stage being set at relatively high torque to prevent stage travel during screening, and the objectives set as follows; $\times 4, \times 10, \times 20, \times 40, \times 100$. The microscopic computer field remained in the off position until the end of the test, to emulate a normal screening microscope. The computer screen was orientated away from the screener. Screeners were informed that the test set of slides were routine cervical smears, and were provided with all relevant clinical data for each slide. The administrator set up the HOME system in the graphics application and entered into the screening mode for the first slide, using the identity code of the volunteer. Screeners were asked to indicate when they had finished each slide and to record a diagnosis on a results summary sheet. At this time the administrator indicated in the graphics application that the screener was leaving the screening mode and set up the system in the screening mode for the next slide. The screener then continued to the next slide and carried on until (s)he had finished the test set.

At the end of the test the HOME system was demonstrated to the screener along with their individual screening performance for each slide.

\subsection{Analysis of data}

Screening time data were exported into an Excel spreadsheet. Slide coverage maps were exported into the Paintbrush application, and edited for printing without altering the relative proportions or size of their components. Slide maps were then superimposed with a map of the coverslip, as recorded 
with the HOME system, and where appropriate were superimposed with a map of the distribution of abnormal cells, as recorded with the HOME system. Slide coverage (area screened in $\mathrm{mm}^{2}$ ) was estimated by dividing the coverage maps into rectangles and calculating the area of each rectangle individually. Final diagnoses were correlated with screening times and coverage. Results are presented as median and range or mean and SD (standard deviation). The significance of any differences was assessed using analysis of variance in order to allow for the effects of different observers and different slides. For analytical purposes the data were transformed logarithmically in order to stabilise the variances. Sensitivity and positive predictive values were calculated according to the Scottish Office report on internal quality control [3].

\section{Results}

\subsection{Study group}

Invitation letters were sent to 29 laboratories in the Thames regions. Six laboratories responded by sending twelve volunteers (eleven female, one male). Experience of screening ranged from three years to twelve years $($ Mean $=6.8 \mathrm{yrs})$. All volunteers except one had passed a competence examination in cervical screening and normally used an Olympus BH2 (6) Leitz laborlux/basic model (4), Gillett and Sibert (1), and Zeiss (1) in their own laboratory. Annual screening workload ranged from 4000-9000 cervical smears per screener.

\subsection{Patterns of screening}

Sixty slides were screened in a horizontal direction, as in Fig. 1(a). Fifty slides were screened in a vertical or "battlement" direction as in Fig. 1(b). Ten slides were screened in a combined horizontal and vertical direction as in Fig. 1(c).

\subsection{Comparison of screening times}

Screening times for all slides are shown in Fig. 2. Mean screening time was $419.6 \mathrm{sec}$ (SD = 227.3). Figure 3 shows screening times for different categories of slide, and mean screening time for negative slides is $451.6 \mathrm{sec}(\mathrm{SD}=221.0)$, mean screening time for abnormal slides is $472.5 \mathrm{sec}$ (SD $=214.2)$, and mean screening time for unsatisfactory slides is $260.3 \mathrm{sec}(\mathrm{SD}=196.8)$. There was no correlation between mean screening time and length of screener experience in cytology.

\subsection{Analysis of slide coverage}

Mean slide overall coverage was $84 \%$. Although some individuals achieved a mean coverage of $99 \%$, none achieved full coverage of every slide. This inter-observer variability is illustrated in Fig. 4. There was no correlation between mean slide coverage and length of screener experience in cytology. The scanning maps shown in Fig. 5(a)-5(e) demonstrate at least five different types of error. Table 1 shows the frequency of different errors, and the mean error rate per slide.

Table 1

Scanning track errors; frequency by type of error

\begin{tabular}{lccccc}
\hline & Underlaps & Retracks & Reversals & HP screen & Early turn \\
\hline Total number & 238 & 133 & 8 & 9 & 99 \\
Mean rate per slide & 1.983333 & 1.108333 & 0.066667 & 0.075 & 0.825 \\
\hline
\end{tabular}




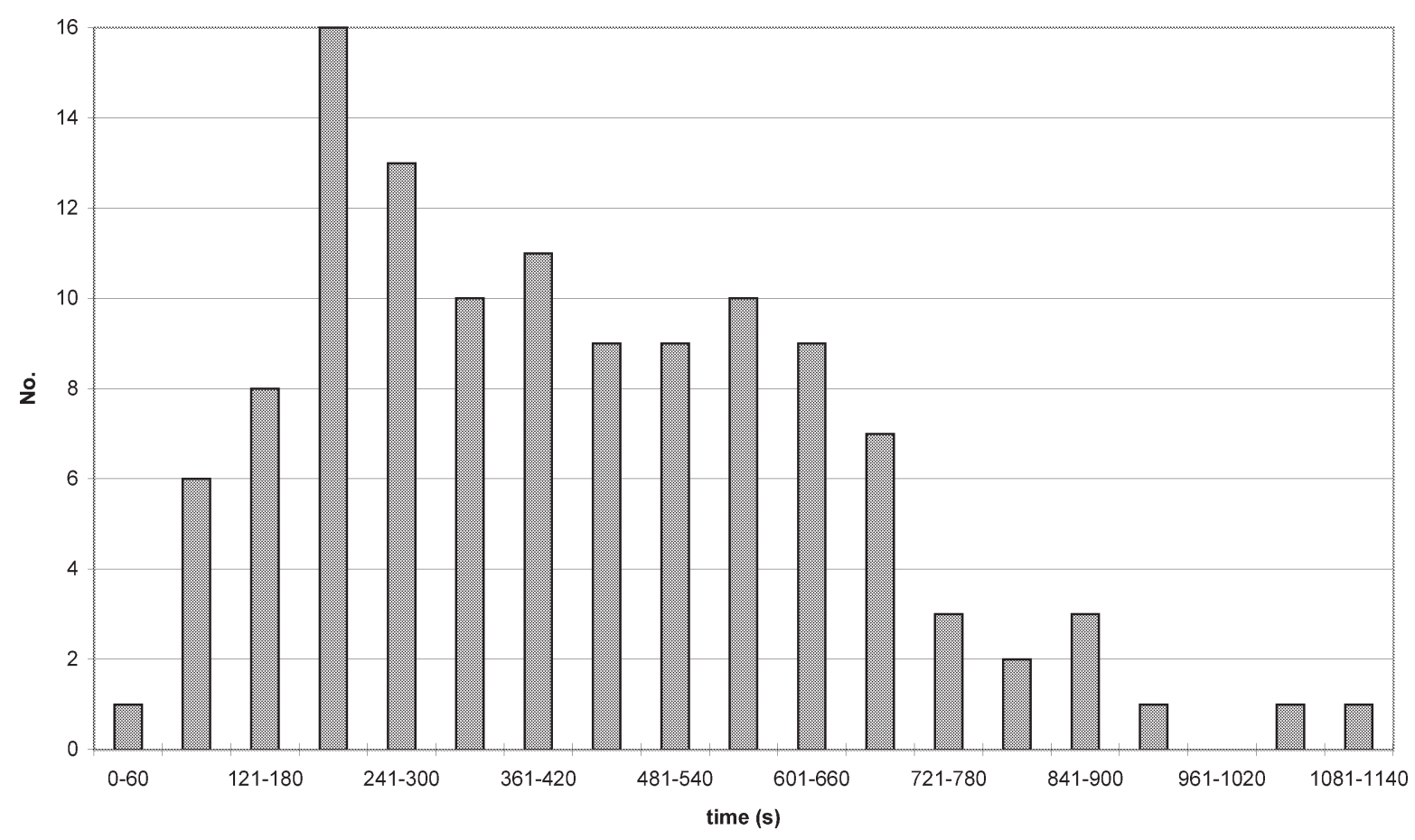

Fig. 2. Primary screening times.

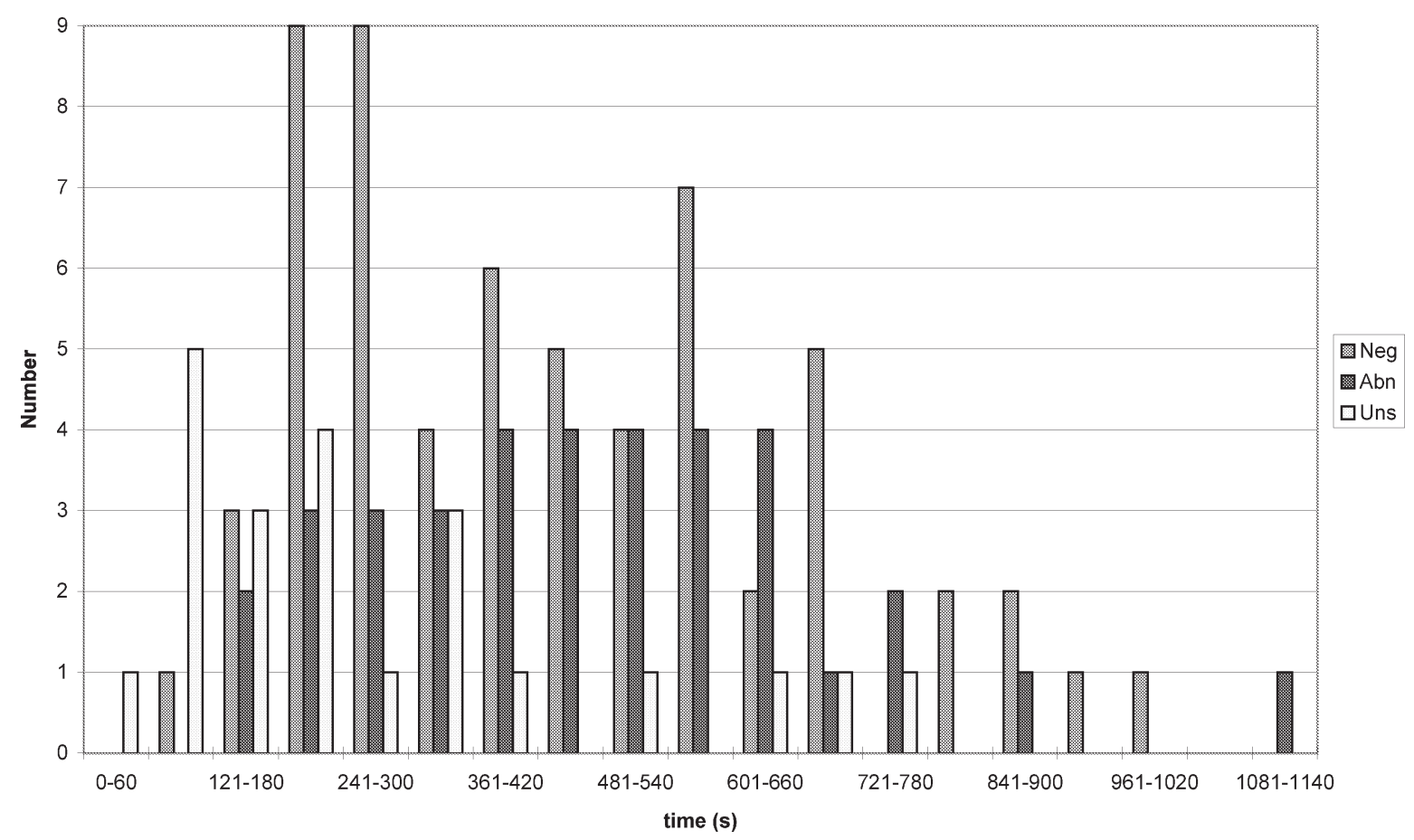

Fig. 3. Primary screening times by diagnosis. 


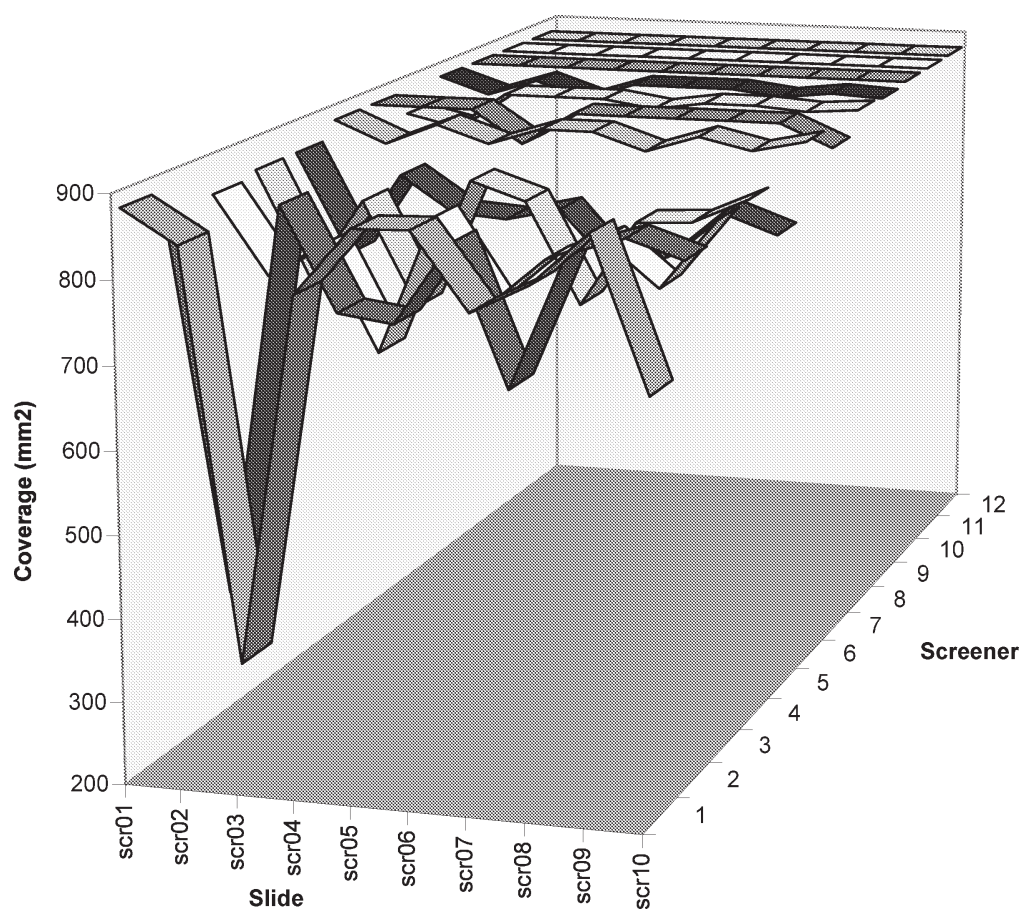

Fig. 4. Slide coverage for individual screeners.

Table 2

Analysis of screener diagnoses

\begin{tabular}{clccccc}
\hline $\begin{array}{c}\text { Slide } \\
\text { no. }\end{array}$ & $\begin{array}{l}\text { Final } \\
\text { diagnosis }\end{array}$ & $\begin{array}{c}\text { No. of correct } \\
\text { diagnoses }\end{array}$ & $\begin{array}{c}\text { False } \\
\text { negatives }\end{array}$ & $\begin{array}{c}\text { False } \\
\text { positives }\end{array}$ & $\begin{array}{c}\text { False } \\
\text { inadequates }\end{array}$ & $\begin{array}{c}\text { Missed } \\
\text { infection }\end{array}$ \\
\hline 01 & Negative & 9 & 0 & 1 & 2 & 0 \\
02 & Positive & 12 & 0 & 0 & 0 & 0 \\
03 & Negative & 12 & 0 & 0 & 0 & 0 \\
04 & Inadequate & 12 & 0 & 0 & 0 & 0 \\
05 & Negative & 10 & 0 & 2 & 0 & 0 \\
06 & Inadequate & 11 & 1 & 0 & 0 & 0 \\
07 & Positive & 12 & 0 & 0 & 0 & 0 \\
08 & Negative (Candida) & 10 & 0 & 1 & 1 & 2 \\
09 & Positive & 11 & 1 & 0 & 0 & 0 \\
10 & Negative (TV) & 4 & 0 & 0 & 8 & 0 \\
\hline
\end{tabular}

\subsection{Sensitivity and positive predictive value}

An analysis of screener diagnosis is shown in Table 2. Correctly identified as being abnormal were 35 of 36 abnormal slides. Sensitivity of the primary screening in this study is therefore $97.2 \%$. The positive predictive value is $89.7 \%$. Sensitivity for infections (Candida albicans and Trichomonas vaginalis) is $91.7 \%$. The slide containing Trichomonas vaginalis was reported as being inadequate by eight screeners. 
a)

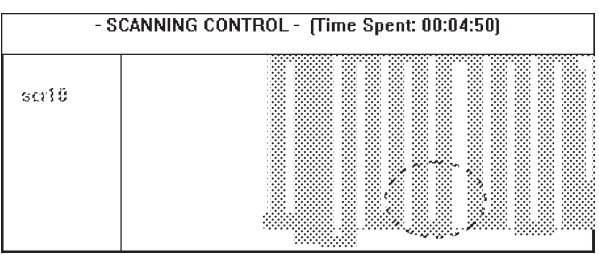

b)

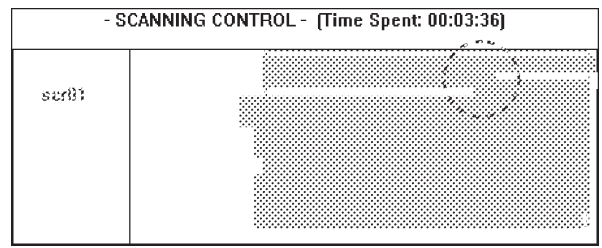

c)

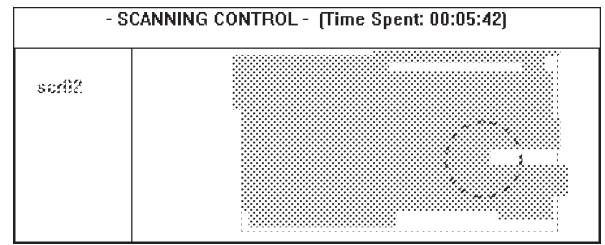

d)

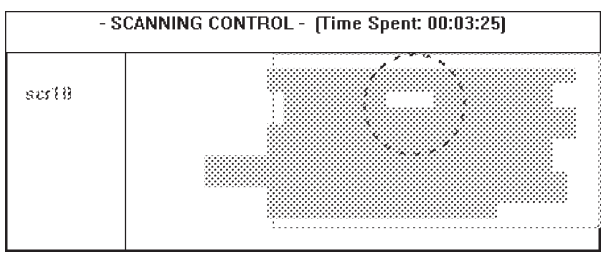

e)

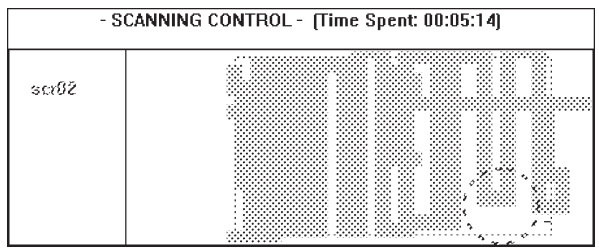

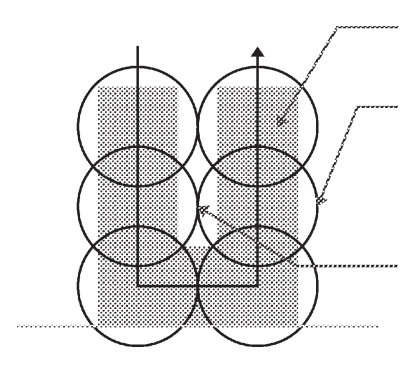

Scanned area

Microscope

field

Area of slide

not sufficiently

covered (white)
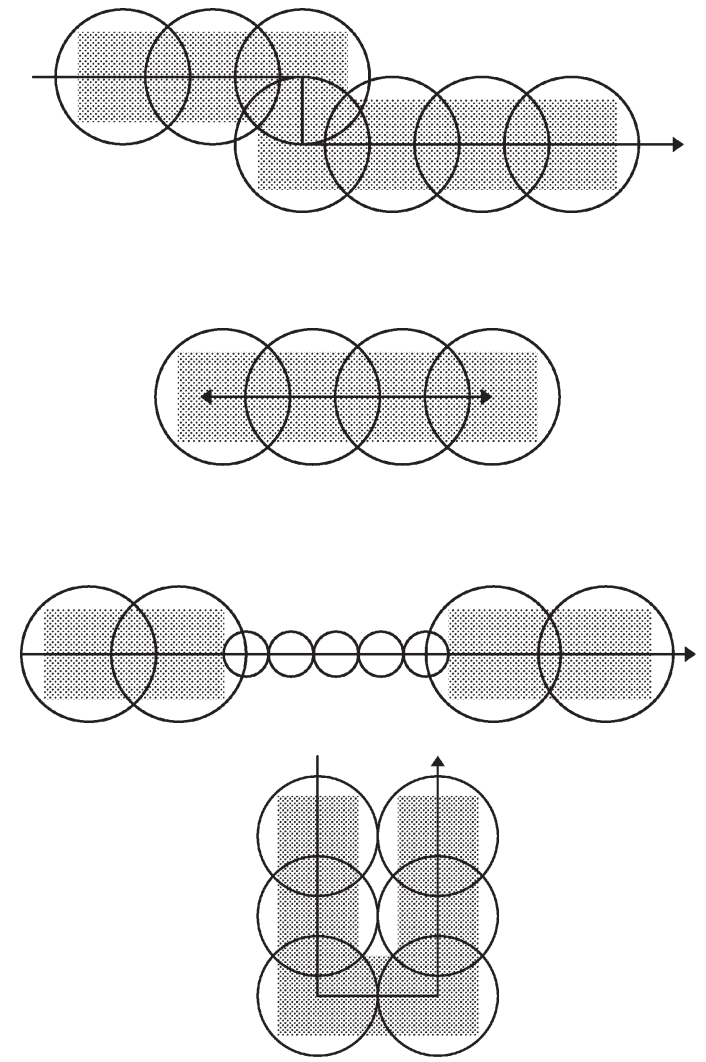

Fig. 5. (a) Insufficient overlap of fields (referred to herein as "underlap"). (b) Failure to retrack scanning track after HP observation at periphery of field (referred to herein as "retrack"). (c) Reversal of screening direction after HP observation (referred to herein as "reversals"). (d) Screening on high power objective (referred to herein as "HP screening"). (e) Screening direction change before edge of coverslip is reached (referred to herein as "early turn") nb. "underlap" also shown here.

\subsection{Analysis of false negative results}

Only one abnormal slide was reported as being negative. The scanning map for this slide is shown in Fig. 6. Analysis of this map shows that abnormal cells were scanned in this case. Screening time for this slide was $184 \mathrm{sec}$ and the area under the coverslip was completely scanned $\left(880 \mathrm{~mm}^{2}\right)$. The mean scanning rate was therefore $4.78 \mathrm{~mm}^{2} \mathrm{~s}^{-1}$ (4.5 fields per sec). No false negative reports were recorded for the group of volunteers who had passed a certificate of competence examination. 


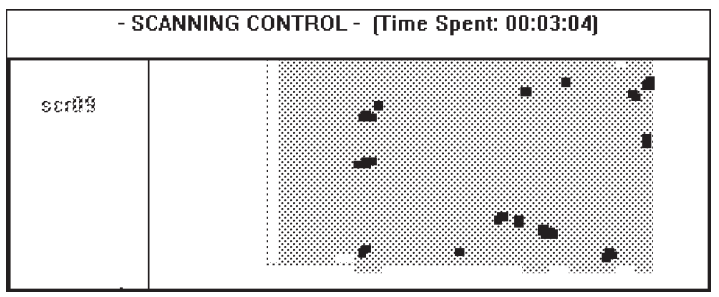

Fig. 6. False negative slide. Abnormal cells shown as black dots.

\section{Discussion}

This study has shown that, although some screeners usually cover the whole slide, none were able to cover the whole of every slide in the test set, and some only covered about half the area under the coverslip. The mean coverage over the study was $84 \%$, and this was not affected by whether horizontal or battlement screening was employed. Within the confines of this study, no relationship between slide coverage and sensitivity was observed, although a more extensive study may be needed to detect such a relationship.

Many reasons for incomplete coverage were observed (see Fig. 3). These included insufficient overlap of fields at the edge of the coverslip, reversal of direction following high power observation, failure to return to the scanning track following high power observation of peripheral cells, and prolonged high power screening. The values for the frequency of these errors may be an underestimate of the true rate in the laboratory environment, where other factors, such as being interrupted during screening, come into play. The significance of the frequency of these errors remains unclear, as they did not affect the diagnostic sensitivity of the slides in this study. However, the frequency of false negative reports caused by screening errors is likely to be extremely low, and would require a much larger sample size to measure it with any accuracy.

These factors were measured by analysis of scanning tracks. The version of the HOME system which we used does not provide data regarding time spent at various powers of magnification, so we were unable to correlate undercoverage as a result of high power screening with frequency of high power observation. Neither could we obtain data regarding the frequency or location of pauses during the screening process, as Schenk and Reuter [4] have measured. This data may have provided more detailed information regarding the reasons for undercoverage.

Sensitivity and positive predictive are both high in this study. This may be a reflection of the extreme care with which volunteers approached the screening of these slides, and may be an inflated value compared to the routine laboratory screening environment. Analysis of the scanning track for the only false negative slide in this study shows that abnormal cells were scanned, but not recognised. The reason for the mis-reporting of this slide cannot therefore be deduced, although the mean scanning rate of 4.5 fields per sec may be too high. Interpretative error cannot be excluded as a reason for this false negative report.

Over-cautious reporting may also account for the majority of screeners reporting slide 10 as inadequate, although this may also reflect variation in reporting of slides with trichomonas and degenerative changes. The authors stand by the reference diagnosis even after review of the case; the presence of some degenerative changes is the most likely explanation for the pattern of reporting, and indicates that the slide was possibly too controversial for inclusion in the study.

This study demonstrates the need for education regarding slide coverage errors, and assessment of their incidence before certificates of competence in the screening of cervical smears are issued. It 
must also be pointed out that if every screener had a HOME Microscope workstation, they would be able to cover the whole of every slide. The coverage patterns seen in Fig. 6 would be very different if this were possible. Although the false negative rate was too low in this study to detect an effect on sensitivity of reporting, significant undercoverage has been demonstrated, and this infers that, given enough time, a screener who is persistently under-covering will miss an abnormality through undercoverage. In order to effectively carry out a task, the screener must be given the right tools for the job; the HOME system constitutes just such a tool.

\section{Acknowledgements}

We would like to thank all the volunteers, without whom the study would not have been possible, and their laboratory managers and consultants for releasing staff to participate, and Anne Morse for providing the slides from St. Mary's NHS Trust cytology slide file and laboratory space for the equipment. Thanks also to Barry Leete of Zeiss UK for technical support with the AxioHOME ${ }^{\circledR}$ system. This research was funded as part of the EC project CYTONET: FILE NO SOC 94/202239 05F02: ITEM B34304.

\section{References}

[1] G. Brugal et al., HOME: Highly Optimised Microscope Environment, Cytometry 13 (1992), 109-116.

[2] A. Morens et al., Tutorial: the HOME Microscope Workstation, Analytical and Quantitative Cytology and Histology 14 (1992), 289-293.

[3] Report of Working Party on Internal Quality Control for Cervical Cytopathology Laboratories, HMSO Scottish Office, 1995.

[4] U. Schenck and B. Reuter, Analysis of the cytoscreening technique as a method of quality control, in: Compendium on Diagnostic Cytology, 7th edn, G. Wied et al., eds, Tutorials of Cytology, Chicago, Illinois, USA, pp. 437-440. 


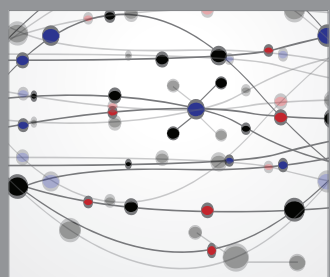

The Scientific World Journal
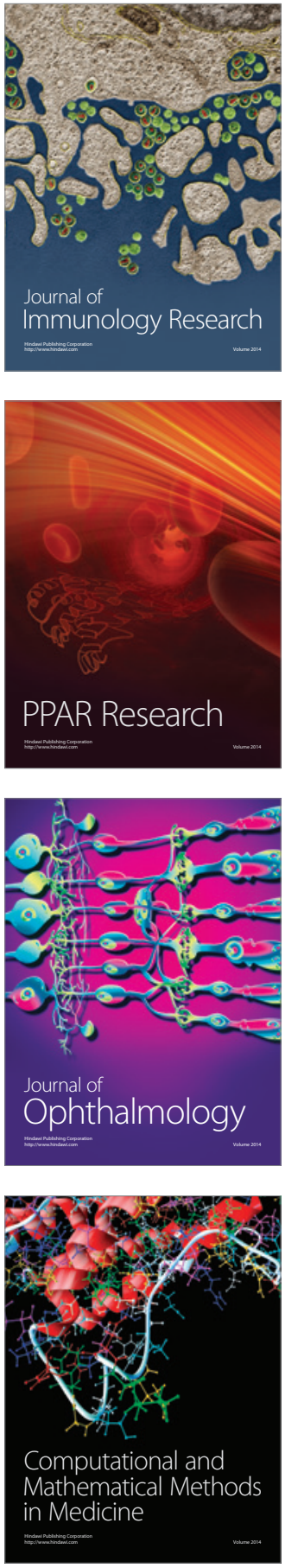

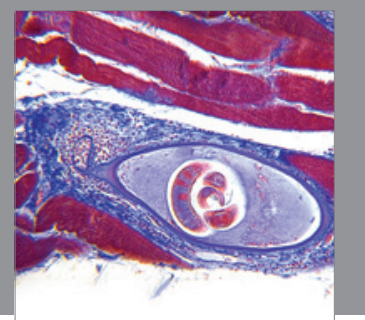

Gastroenterology

Research and Practice
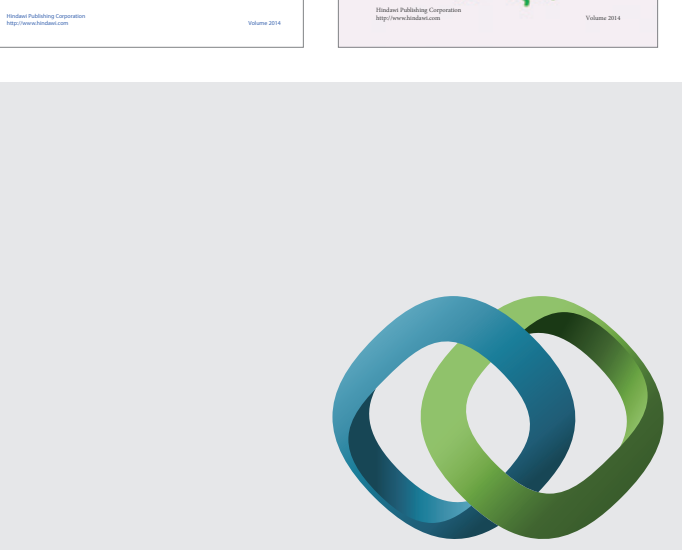

\section{Hindawi}

Submit your manuscripts at

http://www.hindawi.com
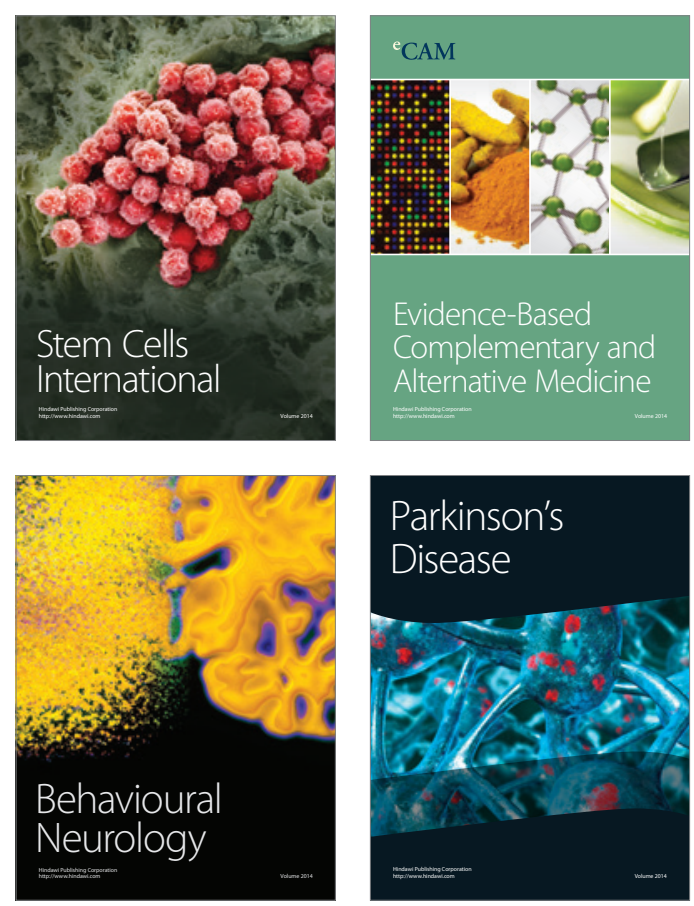

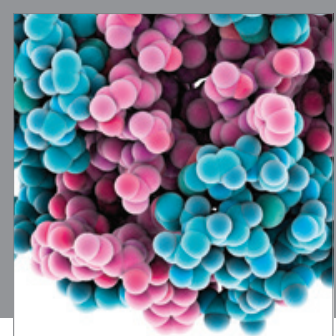

Journal of
Diabetes Research

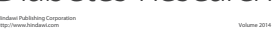

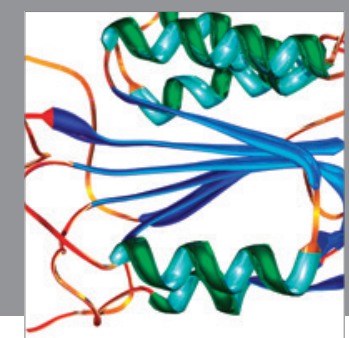

Disease Markers
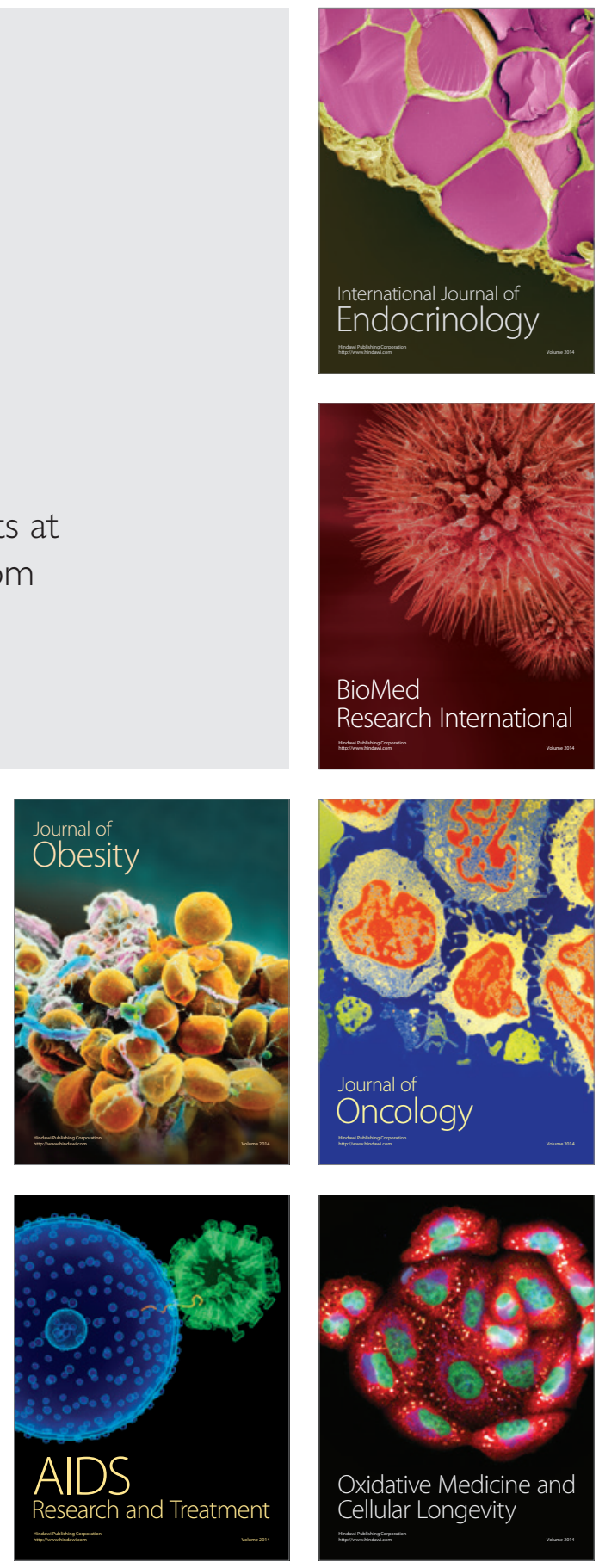